\section{Engaging drug discovery}

\section{By Amy Donner, Senior Editor}

Determining whether a compound binds its intended target is a fundamental question in drug discovery, but assays for measuring target engagement in vivo can be indirect and inconclusive. To remedy the situation, a Swedish group has modified an in vitro assay so that it can be applied to living systems. ${ }^{1}$

The team founded Pelago Bioscience AB to optimize the assay, which they will provide as a service to aid drug discovery programs at client companies, and to adapt the assay for clinical applications.

Current tools that quantify target engagement in vitro include the thermal shift-based assay (TSA), ligand displacement assays, chemoproteomics and tests that look at changes in substrate or product concentrations.

TSAs measure shifts in a protein's thermal stability that occur when bound by a therapeutic. Purified proteins are subjected to
"There is an urgent need for relevant and robust target engagement assays in drug discovery."

-Pär Nordlund, Karolinska Institute
Plotting the quantity of soluble target versus the temperature profile yields a melting curve. As in the in vitro version of the assay, the addition of a therapeutic that stabilizes the target causes the thermal curve to shift.

In proof-of-concept experiments, known binders of four therapeutic target proteins-cyclin dependent kinase 2 (CDK2), CDK6, BRAF and methionine aminopeptidase 2 (MetAP2)triggered shifts in thermal stability. The team tested the approach in lysates, cells and tissues from mice that received the small molecules.

The researchers also used a CETSA with poly(ADP-ribose) polymerase-1 (PARP-1) and showed that olparib but not iniparib induced a thermal shift in the target.

Olparib is a PARP-1 inhibitor from AstraZeneca plc that is in Phase II testing for cancer. In June, Sanofi discontinued development of iniparib after the putative PARP-1 inhibitor failed to meet the primary endpoint of improved overall survival in a Phase III trial. In 2011 and 2012, papers were published showing that iniparib did not bind its target. ${ }^{2,3}$

The CETSA findings were published in Science. The team included scientists from Nanyang Technological University and Linkoeping University.

Nordlund sees CETSAs as a way to "improve the odds for selecting the right targets and validating them for therapeutic development, and to enable companies to select the optimal ligands as clinical candidates."

Brian Hubbard noted that the authors' gradually increasing temperatures in the presence and absence of a bound ligand and are monitored by fluorescence- or light scatteringbased approaches. The extent of temperature shift between the two conditions is proportional to the affinity of the ligand for the target, or the median $\mathrm{IC}_{50}$ value.

Existing in vivo assays for quantifying target engagement have multiple shortcomings.

"There is an urgent need for relevant and robust target engagement assays in drug discovery," said Pär Nordlund, a professor of medical biochemistry and biophysics at the Karolinska Institute and scientific advisor and cofounder of Pelago. "Current assays can be indirect and often dependent on soft biological readouts. This can introduce uncertainties at many steps along the drug discovery process. In extreme cases, compounds have entered clinical trials and later been shown to not even hit their anticipated targets."

To address these problems, a group led by Nordlund and Daniel Molina developed a cellular thermal shift assay (CETSA) for assessing target engagement in vivo. Molina is senior lab manager at Karolinska and CSO at Pelago.

To carry out a CETSA, multiple aliquots of a soluble protein fraction in cell lysates are heated to different temperatures, cooled down and centrifuged to remove any proteins that fall out of solution. The amount of target proteins remaining in solution is quantified by western blotting. experiments do not establish that the assay can be used during candidate optimization because they used compounds that already had been optimized. "During optimization, compounds always have off-target effects. If the CETSA reports no thermal shift, you know the compound isn't binding the target, which is important, but the presence of a thermal shift is not necessarily specific to the target you're interested in."

Hubbard is director of the Therapeutics Projects Group at the Broad Institute of MIT and Harvard and was formerly senior director of cardiovascular diseases at Merck \& Co. Inc. and director of cardiovascular and metabolism research at the Novartis Institutes for BioMedical Research.

Benjamin Cravatt, professor and chair of chemical physiology at The Scripps Research Institute, noted that the assay works only for soluble proteins and will not be useful for membrane proteins, which constitute a sizable portion of therapeutic targets. Protein complexes also could be difficult to use in the assay, he said.

Hubbard was less concerned about the issue of comprehensive coverage. "It is true that there are targets that this assay will simply not work for, but there is no assay that works for everything," he said.

Thus, he sees the clinical application of CETSAs as promising but cautioned that there are multiple remaining hurdles. For example, he said that the assay can reliably report whether or not a drug bound to its target but that it is more difficult to know how much of the 


\section{ANALYSIS}

target was bound.

Cravatt did say that the assay "is probably going to have some real applications because it is easy for people to do. It is much simpler than other kinase assays; no mass spectrometry is needed-just a western blot."

Stefan Knapp, a professor of structural biology at the University of Oxford who has used in vitro TSAs, ${ }^{4}$ also thinks CETSAs could have some useful applications. CETSAs "provide information about drug binding in cells, whereas conventional assays provide information about loss of function of a target. The assay will be very useful for targets for which no specific biomarker exists."

\section{Ready, CETSA, go}

Pelago plans to work with other companies, performing CETSAbased target engagement studies as a service to aid drug discovery efforts. In addition, Pelago is adapting the CETSA for diagnostic and personalized healthcare applications.

"Imagine that large Phase III studies could be conducted and analyzed knowing the exact target engagement status of all enrolled patients. This could help explain poor [responders] and nonresponders," said Nordlund.

Pelago already has data indicating that the method works with serum samples as well as with biopsies from patient tumors. He has filed for a patent covering CETSA as a method to measure ligand binding in complex media. Licenses are available.

Donner, A. SciBX 6(30); doi:10.1038/scibx.2013.778 Published online Aug. 8, 2013

\section{REFERENCES}

1. Molina, D.M. et al. Science; published online July 5, 2013; doi:10.1126/science.1233606

Contact: Pär Nordlund, Karolinska Institute, Stockholm, Sweden e-mail: par.nordlund@ki.se

2. Guha, M. Nat. Biotechnol. 29, 373-374 (2011)

3. Liu, X. et al. Clin. Cancer Res. 18, 510-523 (2012)

4. Fedorov, O. et al. Proc. Natl. Acad. Sci. USA 104, 20523-20528 (2007)

COMPANIES AND INSTITUTIONS MENTIONED

AstraZeneca plc (LSE:AZN; NYSE:AZN), London, U.K. Broad Institute of MIT and Harvard, Cambridge, Mass.

Karolinska Institute, Stockholm, Sweden

Linkoeping University, Linkoeping, Sweden

Merck \& Co. Inc. (NYSE:MRK), Whitehouse Station, N.J.

Nanyang Technological University, Singapore

Novartis Institutes for BioMedical Research, Boston, Mass.

Pelago Bioscience AB, Stockholm, Sweden

Sanofi (Euronext:SAN; NYSE:SNY), Paris, France

The Scripps Research Institute, La Jolla, Calif. University of Oxford, Oxford, U.K. 